\title{
Effectiveness of Acupressure, Acupuncture and Buerger's Exercises in Varicose Veins: A Case Study
}

\author{
Authors \\ Thakrar J.V ${ }^{1 *}$, Sakshi Thakrar ${ }^{2}$, Khatri S.M ${ }^{3}$ \\ ${ }^{1,2}$ Assistant Professor, ${ }^{3}$ Principal \\ Nootan College of Physiotherapy, Sankalchand Patel University, Visnagar, Gujarat, India -384 315 \\ *Corresponding Author \\ Thakrar J.V \\ Email: jayeshthakrar2107@gmail.com
}

\begin{abstract}
Varicose veins are widened superficial veins that commonly affect lower limbs and are easily visible; therefore it is also a cosmetic issue for large proportion of people, especially during warm weather symptom scan include heaviness, stiffness, itching, pain and cramps. In this study along with Buerger's exercise, acupressure and acupuncture with stimulation (at point GB 34, ST 44) was applied as additional interventions in female patient of age 47 years for eight treatment sessions. We found promising results of the combined interventions in this single case.
\end{abstract}

Keywords: varicose veins, acupressure, acupuncture, Buerger's exercise, visual analogue scale.

\section{Introduction}

Varicose veins are commonly affecting lower limbs.

Although, mostly it a cosmetic issue along with symptoms like heaviness, tightness, itching, pain and cramps especially during warm weather. It is seen in $10 \%$ percent of the general populace, during physical examination it is observed as abnormal dilated and tortuous veins. ${ }^{1,2}$

In our country, the number of people with this disorder is rising exponentially and the extrapolated prevalence rate of varicose vein in India is about $47,928,177$. According to calculation, percentage of sufferers in India from vein disease was $15-20 \% .^{3}$
Up till now, clinical research in venous ulcer disease has concentrated mainly on circulatory or wound care issues rather than physical functioning of the individual. Due to physical inactivity and ankle stiffness patients frequently enter a nonhealing state and until the mid-1990s medical literature rarely focused on mobility and quality-oflife topics in chronic leg ulcers. Above all, the potential benefit of comprehensive advice and physical rehabilitation in this disease category has not been thoroughly studied. ${ }^{4}$

Complimentary therapy or alternative therapy can be an important linkage between varicose vein 
victim, physiotherapy and medical management. Complimentary therapy is often considered by patient for various reasons that includes relief of symptoms, reducing side effects, feeling more control, natural therapy, natural healing, comfort from touch, talk and tie, boosting immune system etc. $^{5}$

Traditional Chinese Medicine interventions like acupressure and acupuncture on regular basis is believed to benefit the patients with varicose veins and can be a huge help for the physical and emotional discomfort associated with this disorder. ${ }^{6}$

Due to chronic and severity of health problem in these patients, wound care providers and physiotherapists are attracted to help and work at modest level so as to treat and relieve them. The aim of the present study was to discuss regarding disease, its effects and remedy in elderly females with chronic leg ulceration which is due to lack of proper guidance, precaution and treatment in primary stages of varicose vein. Also evaluation was done among same age groups for forth coming physiotherapy approaches ${ }^{7}$

\section{Cases Study}

\section{History}

We describe a 47 year old female patient with height of 165 centimeters, weight 70 kilograms and body mass index (BMI) was 25.71. She was married, mother of 2 children and was teacher by profession. The patient was seen by a physiotherapist and enrolled for daily treatment. She was referred for physiotherapy by a general practioner and diagnosed as varicose veins in right leg. She complained of pain and cramps in leg especially during night since more than six months. She thought her discomfort was more and attributed to standing and exertion due to her job and household works.

\section{Physical examination}

During examination vital signs were normal and increase in lumbar lordosis was observed in postural findings. On inspection, prominent dark blue veins, swelling in right leg, darkening of skin in affected leg was seen. Palpation of varicose vein was done in prone lying and her highest discomfort during her work was noted as 8/10 on NPRS scale.

\section{Procedure}

Ethical approval was granted from the Institutional Ethical Committee and the patient gave an informed written consent. Her demographic data, physical examination, and NPRS score was recorded. Then she was treated with acupressure therapy, acupuncture treatment and Buerger's exercises. For acupressure therapy, patient was asked to sit in chair comfortably. Procedure for acupressure was briefly explained to gain patient's cooperation. After counseling patient, mild pressure for three minutes was given at point number 8 (Figure 1) on thenar surface of hand. Point no 8 represents an area for thyroid and parathyroid which is believed to be effective for cramps. For acupuncture therapy, patient was informed about this therapy then skin area was cleaned with spirit gauze then patient was asked to be in supine position with knee flexed to 15-20 degree. Point no. ST 44 (Figure 2) ${ }^{8}$ G.B 34 (Figure 3 ) were selected ${ }^{9}$ needle of size1 tsun for G.B 34; 0.5 tsun for ST 44 (Figure 4) was used. Acupuncture stimulator (Figure 5) was used to stimulate acupuncture points via needles of respective sizes. Both points were stimulated simultaneously by connecting needles with stimulator and duration of treatment was to 8 minutes. This treatment was continued for eight days on daily basis and the outcome in terms was NPRS was 2/10 after the final treatment. 


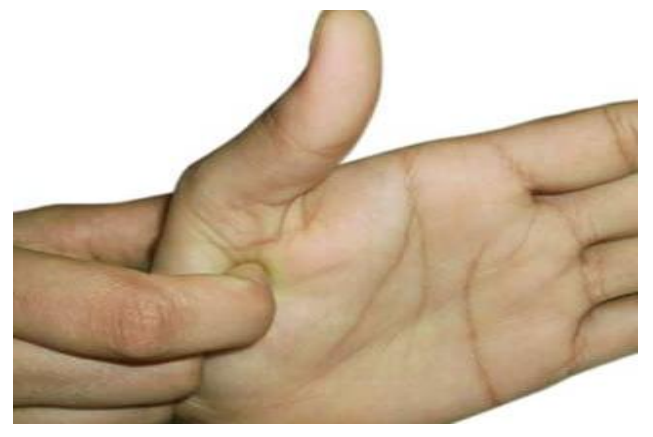

Figure 1. Point number 8 , Thenar surface

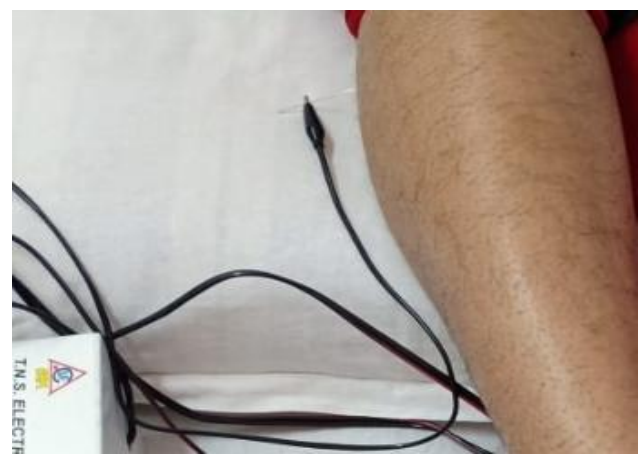

Figure 3. Point no. G.B 34

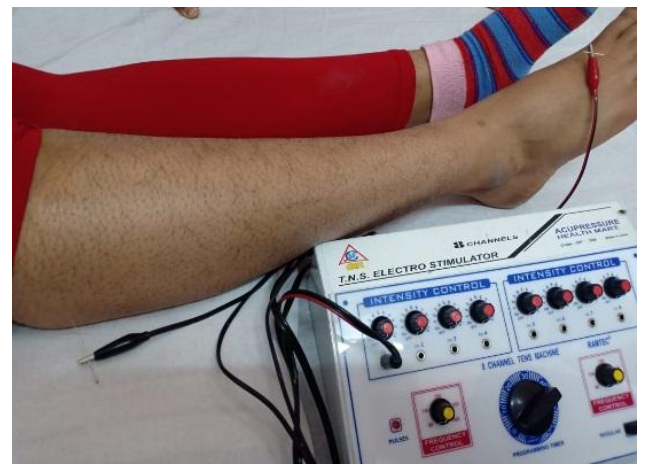

Figure 5: Acupuncture stimulation at point GB 34, ST44

\section{Discussion}

This case discusses management of varicose veins by physiotherapy interventions along with acupressure and acupuncture. Some evidences are available which supports utility of Beurgers exercises. It also proposes that Buerger's exercises might be substitute procedure for improving peripheral circulation, but still there is requirement for more advanced investigation and interventions along with Buerger's exercises ${ }^{10}$.Further, significant

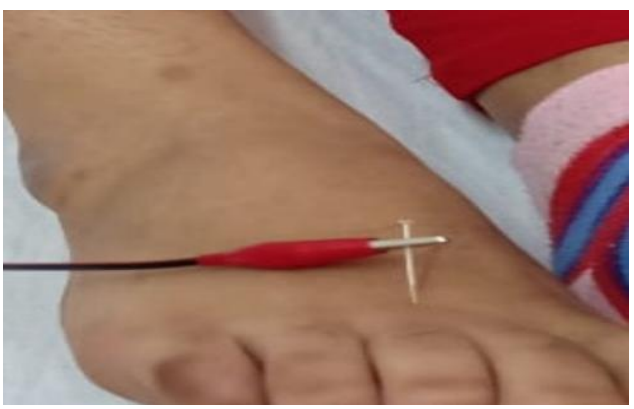

Figure 2. Point no. ST 44

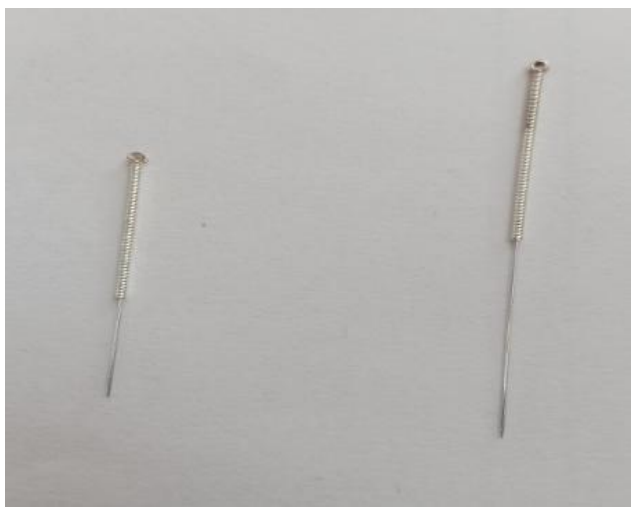

Figure 4. Needle Size 0.5 tsun, 1 tsun relief in symptoms were noted in the present case. This could be due to the combined physiotherapy interventions, effectiveness of acupressure by enhancing lymphatic drainage and acupuncture through neuroharmonal pathways. ${ }^{11.12}$

\section{Conclusion}

Combination therapy in form of conventional physiotherapy intervention like Buerger's exercises along with acupressure and acupuncture can be used 
in the management of patient with varicose veins so as to minimize the physical and emotional discomfort.

\section{References}

1. Bruce Campbell, Clinical review. Varicose vein and their management, BMJ volume 333, 5 august 2006; 287.

2. Mulla SA et al, International Surgery Journal 2017 Feb; 4(2):529-533.

3. Nayala Diwan, Global journal for research analysis, Efficacy of Buerger's exercise versus faradism under pressure in varicose veins.Volume-7, issue-3, march-2018.

4. Gregory Piazza, Clinician Update, Varicose veins, 2014; 130:582-587.

5. Christian Nordqvist; Medical news today, How does acupuncture works? Thu 21 December

2017.https://www.medicalnewstoday.com/ar ticles/156488.

6. Vanessa Vogel, Batt, L.AcMSTOM, Is Acupuncture Good for Varicose Veins? https://www.acufinder.com/Acupuncture+In formation/Detail/Is+Acupuncture+Good+for +Varicose+Veins+ [accessed Jan 13 2019]

7. Kirsti Skavberg Roaldsen, Physiotherapy Research International, Functional ability in female leg ulcer patients - a challenge for physiotherapy. December 2006 11(4):191203

8. Traditional Chinese Medicine Points, https://tcmpoints.com/stomach/st44neiting/[accessed 12 Jan 2019]

9. Acupuncture technology news, https://www.miridiatech.com/news/2016/05/ acupuncturepoint-gallbladder-34/ [accessed 21 Jan 2019]
10. Chyong-Fang Chang1, Chang-Cheng Chang2, Mei-Yen Chen1.Open Journal of Nursing, 2015, 5, 120-128.

11. Qian-Qian Li, Guang-Xia Shi, Qian Xu, Jing Wang, Cun-Zhi Liu and Lin-Peng Wang, Evid Based Complement Alternat Med, NCBI, Acupuncture Effect and Central Autonomic Regulation,2013 May26.

12. Robert A. Charman, FCSP DipTP, Physiotherapy journal Acupressure, July 2001 Volume 87, Issue 7, 383. 